\title{
RESEARCH REPORT \\ PEMF fails to enhance nerve regeneration after sciatic nerve crush lesion
}

\author{
Abrahão Fontes Baptista ${ }^{1,2,3}$, Bruno Teixeira Goes ${ }^{4}$, Diego Menezes ${ }^{3}$, Flávia Carvalho \\ Alcantara Gomes ${ }^{2}$, João Zugaib ${ }^{4}$, Joice Stipursky ${ }^{2}$, Joyce R. S. Gomes ${ }^{4}$, Júlia \\ Teixeira Oliveira ${ }^{2,4}$, Marcos André Vannier-Santos ${ }^{3}$, and Ana Maria Blanco Martinez ${ }^{2}$ \\ ${ }^{1}$ Universidade Federal da Bahia, Biomorphology Department, Health Sciences Institute, Salvador, BA; ${ }^{2}$ Universidade Federal \\ do Rio de Janeiro, Biomedical Sciences Institute, Health Sciences Center, Rio de Janeiro, RJ; ${ }^{3}$ Fundação Oswaldo Cruz, \\ Gonçalo Moniz Research Center, Electron Microscopy Unit, Salvador, BA; and ${ }^{4}$ Escola Baiana de Medicina e Saúde Pública, \\ Neuromusculoskeletal Dynamics Research Group, Salvador, BA, Brazil
}

\begin{abstract}
The use of electromagnetic fields has been reported to enhance peripheral nerve regeneration. This study aimed to identify the effects of a prolonged protocol of low-frequency pulsed electromagnetic field (PEMF) on peripheral nerve regeneration. Thirty-four male Swiss mice (Mus musculus) were divided into PEMF ( $n=17$ ) and control $(n=17)$ groups. All animals underwent a unilateral sciatic-crush lesion, and the PEMF group was exposed to a $72-\mathrm{Hz}, 2-\mathrm{G}$ electromagnetic field for $30 \mathrm{~min}$, five days a week, for three weeks. Functional analysis was carried out weekly. After three weeks, the animals were euthanized, and histological, morphometric, oxidative stress, and TGF- $\beta 1$ analyses were performed. Functional analysis showed no differences between the groups. Histological appearance was similar between PEMF and control nerves. Morphometric assessment showed that the PEMF nerves trended toward decreased regeneration. The levels of free radicals were more pronounced in PEMF nerves, but were not associated with an increase in the content of the TGF- $\beta 1 / \mathrm{Smad}$ signaling pathway. Prolonged PEMF regimen leads to delayed histological peripheral nerve regeneration and increased oxidative stress but no loss of function recovery.
\end{abstract}

Key words: electromagnetic fields, mouse, regeneration, sciatic nerve lesion

\section{Introduction}

The influence of low-frequency pulsed electromagnetic fields (PEMF) on peripheral nerve regeneration in experimental animals has been studied using a variety of protocols, with amplitudes generally between 0.3 and $300 \mathrm{mT}$, and frequencies between 2 and $1,000 \mathrm{~Hz}$ (Sisken et al., 1989; Rusovan et al., 1992; Kanje et al.,

\footnotetext{
Address correspondence to: Prof. Ana Maria Blanco Martinez, Departamento de Histologia e Embriologia, Centro de Ciências da Saúde, bloco F, sala F012, Ilha do Fundão, Rio de Janeiro, RJ, CEP 21.949-900, Brazil. Tel: +(55)21-2562-6431; Fax: +(55)21-2562-6431; E-mail: martinez@histo.ufrj.br
}

1993; Ottani et al., 1998; Kim et al., 2002; Bervar, 2005). The mechanism of action is reported to be related to ionic mobilization through cell membranes, enhancing the permeability to ions such as calcium, and triggering intracellular signaling cascades that can initiate, facilitate, or inhibit various biological processes (Balcavage et al., 1996; Oschman, 2004), possibly acting on the regeneration process. PEMF may also affect Wallerian degeneration (WD), stimulating protein synthesis (Sisken et al., 1989) and enhancing local concentrations of growth factors such as transforming growth factor- $\beta 1$ (TGF- $\beta 1$ ). This increase in the TGF- $\beta 1$ level will induce neuroglial signals, modulate Schwann cell (SC) activity (Rogister et al., 1993), and direct 
neurite growth (Macias et al., 2000). TGF- $\beta 1$ induces proliferation of SCs in culture, promoting a pre- or nonmyelinating phenotype. However, when neurons are in contact with SCs, TGF- $\beta 1$ inhibits their proliferation and axon myelinization (Einheber et al., 1995), by blocking the expression of myelin-related molecules (Guénard et al., 1995). Acting as a multifunctional regulator, TGF$\beta 1$ could improve regeneration after a peripheral nerve lesion, promoting the early differentiation of SCs, but impair nerve-fiber maturation in late phases, when growth cones have already reached the target organs and their main activity regresses from regeneration to nerve-impulse conduction. Therefore, brief periods of PEMF after peripheral nerve lesions could be beneficial, but prolonged use could lead to inhibition of the regeneration process. This may probably occur through an oxidative stress pathway, which activates TGF- $\beta 1$ and transglutaminase enzyme (TGase2) in the intracellular environment, causing nuclear translocation of Smad3, and, as a consequence, protein aggregation (Lafon et al., 1996; Hong et al., 1997; Koo et al., 1999; Junn et al., 2000; Chiu et al., 2001; Herrera et al., 2001a; 2001b; Jiang et al., 2003; Rhyu et al., 2005; Shin et al., 2008).

Since many studies have used brief protocols of PEMF to study peripheral nerve regeneration, we performed an exploratory study to analyze the influence of a prolonged PEMF protocol on this process. We evaluated the influence of PEMF on oxygen-reactive species formation after the end of the treatment period and its possible relationship to expression of TGF- $\beta 1$.

\section{Materials and Methods}

This experimental study was approved by the Ethics Committee for Animal Use of the Bahian Medicine and Public Health School. We used 34 male Swiss mice (Mus musculus), weighing 30-45 g, housed in individual cages, with food and water ad libitum, and a 12/12 h light/dark cycle.

\section{Surgical procedures}

The animals were deeply anesthetized with ketamine $(0.1 \mathrm{ml} / \mathrm{mg})$ and xylazine $(0.2 \mathrm{ml} / \mathrm{mg})$, and then the sciatic nerve was exposed, isolated from the adjacent tissues, and crushed $1 \mathrm{~cm}$ from the spinal cord by means of a non-serrated needle holder, maintained on the first lock for $30 \mathrm{~s}$.

\section{PEMF stimulation}

PEMF stimulation was initiated two days after surgery. Animals were lightly anesthetized with halothane using a vaporization system (Takaoka, USA) together with oxygen $(1 \mathrm{l} / \mathrm{min})$. The PEMF group ( $n=17)$ was stimulated with a device generating $72-$ $\mathrm{Hz}, 2-\mathrm{G}$, pulse width $<200 \mathrm{~ns}$ and ramp time less than $10 \mathrm{~ns}(\mathrm{~dB} / \mathrm{dt}$ ) (SOLO-EM PROBE Technologies, USA) positioned above the skin, on the lesion site, for 30 min, five days a week, for three weeks. The control group ( $n=17$ ) was submitted to the same conditions as the PEMF group, but without the electromagnetic field. Normal nerves were taken from the contralateral side (left), which was not submitted to the crush lesion and did not receive the PEMF dispositive above them.

\section{Functional assessment}

Functional assessment was performed on the day prior to the surgery and weekly on the 7th, 14th, and 21 st days post-lesion, by means of the Sciatic Static Index for mice (SSIm), according to our previously described protocol (Baptista et al., 2007). In the SSIm, 0 corresponds to normal function (i.e., both sides with the same functional status), and -100 corresponds to a total functional impairment of one side.

\section{Histomorphometric assessment}

On the 21st day post-lesion, after functional assessment, 10 animals (PEMF, $n=5$; Control, $n=5)$ were deeply anesthetized with ketamine and xylazine and euthanized by transcardiac perfusion with a fixative solution (4\% paraformaldehyde and $2 \%$ glutaraldehyde in $0.1 \mathrm{M}$ sodium cacodylate buffer, $\mathrm{pH} 7.4,25 \mathrm{ml} / \mathrm{animal})$. The sciatic nerves ipsilateral to the lesion were harvested, and a 2-mm segment, $3 \mathrm{~mm}$ distal from the lesion site, was dissected. Contralateral nerves (normal nerves, $n=10$ ) were also dissected, and a 2-mm segment was collected from the equivalent portion of the lesioned nerve.

The segments were post-fixed, osmicated, dehydrated in increasing concentrations of acetone (30-100\%), infiltrated, and plastic embedded. Transverse sections of $0.5 \mu \mathrm{m}$ (semithin) and $70 \mathrm{\eta m}$ (ultrathin) were obtained using an ultramicrotome (Ultracut, Reichert-Jung). The semithin sections were stained with $1 \%$ toluidine blue. Images were acquired on a light microscope (Olympus CX41) connected to a digital camera (Evolution Color PL 1642, Media Cybernetics). A $1,000 \times$ magnification was used to assess the general morphological condition of the nerves and the number and area of blood vessels.

For the ultrastructural analysis, $70 \eta \mathrm{m}$ sections were obtained, contrasted with 1\% uranyl acetate and lead citrate, and observed through a transmission electron microscope (Zeiss EM 109) equipped with an image acquisition system (MegaView II, AnalysisImaging System). A magnification of 7,000x was used for morphological assessment and measurement of the densities and diameters of myelinated and 
non-myelinated fibers, as well as the number of SC nuclei. Densities were calculated by dividing the number of cells by the total area of 10 systematically chosen fields. Using these measurements, the $g$ ratios (axon diameter/fiber diameter) were also obtained.

\section{Oxidative stress analysis}

Biochemical analysis of oxidative stress was also accomplished after the last day of functional analysis, using 14 animals. Seven PEMF animals and seven controls were allocated to the analysis of reactiveoxygen species formation, by the TBARS method [reactive substances to thiobarbituric acid (TBA)], which is based on the level of lipid peroxidation (Menezes et al., 2006; Vannier-Santos et al., 2008). After the mice were euthanized, similar segments from both sciatic nerves were dissected as described previously, washed in saline solution buffered with sodium phosphate (PBS), and resuspended in $200 \mu \mathrm{l}$ of the same solution to which was added $200 \mu$ l of $1 \%$ TBA. Subsequently, the samples were incubated at between 97 and $100^{\circ} \mathrm{C}$ for $2.5 \mathrm{~h}$ to obtain the colorimetric assay. TBA-reactive substances were read on a spectrophotometer (Hitachi U-1100) at $532 \mathrm{~nm}$.

\section{Immunohistochemistry}

Four animals (PEMF, $n=2$; Control, $n=2$ ) were transcardially perfused in the same manner as previously described. Nerves were removed, cryoprotected by incubation in a sucrose gradient $(10-30 \%)$, and embedded in O.C.T. (Tissue-Tek) for frozen sectioning in a cryostat. The $10-\mathrm{mm}$ sections obtained were washed in PBS and subsequently incubated for $1 \mathrm{~h}$ in $3 \%$ BSA, $5 \%$ NGS, and $0.3 \%$ Triton X-100 at room temperature. After that, the sections were incubated with primary antibodies diluted in the same solution without Triton X-100 (blocking solution), overnight at $4^{\circ} \mathrm{C}$. Then, sections were washed with PBS and incubated with secondary antibodies for $2 \mathrm{~h}$ at room temperature. Primary antibodies were mouse anti-TGF- $\beta 1$ (R\&D Systems, 1:100), rabbit anti-TGFRII (Santa Cruz Biotechnology, 1:100), rabbit anti-phospho Smad2/3 (Santa Cruz Biotechnology, 1:100), and mouse anti-Smad4 (Santa Cruz Biotechnology, 1:5). Secondary antibodies were all from Molecular Probes: goat anti-mouse IgG conjugated with Alexa fluor 488 (1:300), goat antirabbit IgG conjugated with Alexa fluor 546 (1:1000), and goat anti-mouse IgG conjugated with Alexa fluor 546 (1:1000). Nuclei were counterstained with DAPI (Sigma Chemical Co.) or TO-PRO (Invitrogen). Negative controls were established by omitting the primary antibody during staining. In all cases, no reactivity was observed when the primary antibody was absent. Cell preparations were mounted directly on $\mathrm{N}$-propyl gallate and observed by means of a TE300 Nikon Microscope.

\section{Statistical analysis}

The independent variable for all the groups was the influence of the PEMF. The dependent variables were derived from functional, histomorphometric, biochemical, and immunohistochemical analyses. In the functional analysis, using the SSIm, we performed paired (intragroup, between days) and unpaired (between groups, on each day) analyses. For histomorphometric analysis, we considered as dependent variables the number and area of blood vessels (obtained at 1,000x magnification), density and diameter of myelinated fibers stratified in 0-2, 2-6 and $6-12 \mu \mathrm{m}$, density and diameter of non-myelinated fibers, density of SC nuclei, and $g$ ratio (obtained at $7,000 \times$ magnification) stratified in ranges of $0.1-0.4$, $0.4-0.5,0.5-0.6,0.6-0.7$, and $0.7-0.9$. When ranges were used, the lowest portions were always included and the highest portions excluded (e.g., the 0-2 range includes $0-1.99$, excluding 2). Paired inferences were done by the Friedman non-parametric test, and unpaired inferences by the Kruskal-Wallis nonparametric test, together with the Student-NewmannKeuls post-hoc analysis, when necessary. The $\mathrm{Cl}$ was $95 \%$, with an accepted alpha value of $5 \%(p<0.05)$. The analyses were carried out using the BioEstat 5.0 statistical software (Mamirauá, Brazil).

\section{Results}

\section{Functional assessment}

There were no signs of functional impairment on the last day of the treatment period. Both groups displayed a similar pattern during the entire assessment period, as demonstrated by the SSIm. In the first week post-lesion, the animals showed the most pronounced loss of function, but recovered gradually after this time. Paired analysis by the Friedman non-parametric test showed significant differences between weeks for the PEMF ( $F r=29.75$; $\mathrm{p}<0.0001)$ and control $(\mathrm{Fr}=25.73 ; \mathrm{p}<0.0001)$ groups, but unpaired analysis, comparing the groups on each day of functional assessment, did not show significant differences.

\section{Morphological assessment}

Morphological abnormalities were assessed by both light and electron microscopy. By light microscopy, both crushed and stimulated (PEMF) and control nerves showed, in general, fibers with smaller calibers, larger endoneurial spaces, and sometimes the presence of axons with dark axoplasm when compared 
to normal nerves (Fig. 1). We did not find signs of edema in any of the specimens analyzed.

Electron microscopy showed that PEMF and control nerves were similar, with a predominance of small-diameter myelinated fibers and the presence
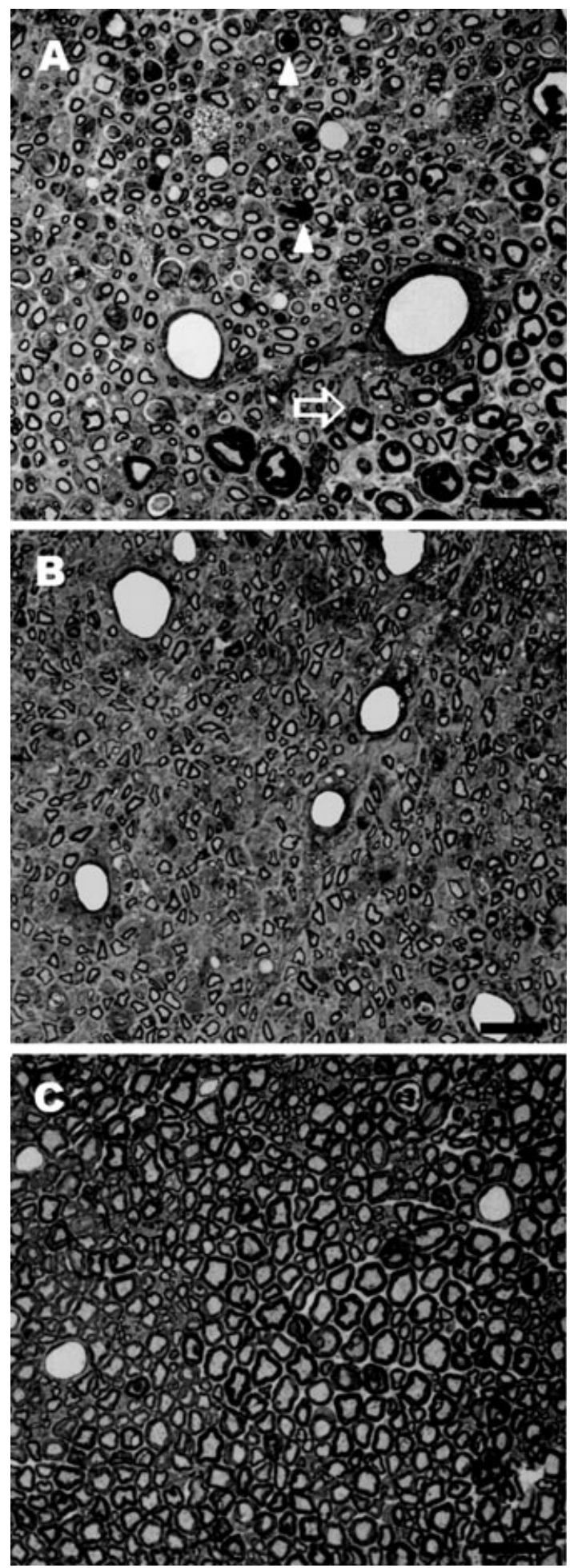

of regenerative islands, lipid inclusions, and myelin figures in both conditions. There were nerve fibers in the initial stage of remyelination, characterized by a very thin myelin sheath (Fig. $2 \mathrm{~A}$ and B). Normal nerves showed higher-caliber myelinated fibers, with a well-developed myelin sheath, compact grouping of unmyelinated fibers, and normal-appearing endoneurial space (Fig. 2C).

\section{Histomorphometry}

In morphometric analysis by light microscopy, the density of blood vessels was similar in the three groups of nerves, but more variable in the controls. Although not significantly different, nerves stimulated with PEMF and normal nerves showed larger-diameter blood vessels than the controls (Fig. 1).

Assessment of myelinated fibers, stratified by diameter, showed that density, fiber and axon caliber, and myelin area were similar between the three groups in the range of $0-2 \mu \mathrm{m}$ diameter (Fig. 3A, D, $\mathrm{G}$, and J). The majority of fibers were concentrated in the range of 2-6 $\mu \mathrm{m}$ in all groups. PEMF-stimulated nerves contained significantly more myelinated fibers in this range, when compared to normal nerves $(p<0.05)$, but there were no significant differences between PEMF and control nerves (Fig. 3B). In the 6-12 $\mu \mathrm{m}$ diameter range, PEMF and control nerves contained significantly fewer fibers than normal nerves $(p \geq 0.05)$ (Fig. 3C). Regarding myelinated fiber and axon diameters, PEMF nerves and controls contained significantly smaller fibers than normal nerves, again in the $6-12 \mu \mathrm{m}$ range (Fig. 3F and I). Myelin area was decreased in crushed nerves (PEMF and control) in the range of 2-6 $\mu \mathrm{m}$ (Fig. 3K). However, in the range of 6-12 $\mu \mathrm{m}$ diameter, only PEMF nerves had significantly less myelin than normal ones (Fig. 3L). This pattern of myelination matched with the $g$ coefficient analysis. The majority of myelinated fibers, from normal and control nerves, showed $g$ coefficients in the range of 0.6-0.7, while nerves stimulated with PEMF had most of their fibers in the range of 0.7-0.9 (Fig. 4). The

Figure 1. Semithin transverse sciatic nerve sections. Images correspond to sections of an area $3 \mathrm{~mm}$ distal to the crush lesion, three weeks after surgery. (A) PEMF nerve (crush lesion and treatment with a $72-\mathrm{Hz}$ electromagnetic field), showing mainly grouped low-diameter myelinated fibers (arrow). Some fibers with dark axoplasm were seen (arrow heads), and also increased endoneurial space and blood vessels with various calibers. (B) Control nerve (crush lesion, without treatment), also presenting low-diameter myelinated fibers and increased endoneurial space. Blood vessels were constantly seen, but fibers with dark axoplasm were less incident. (C) Normal nerve (without lesion of stimulation), showing a normal pattern of fiber morphology and distribution. Scale bar $=10 \mu \mathrm{m}$. 

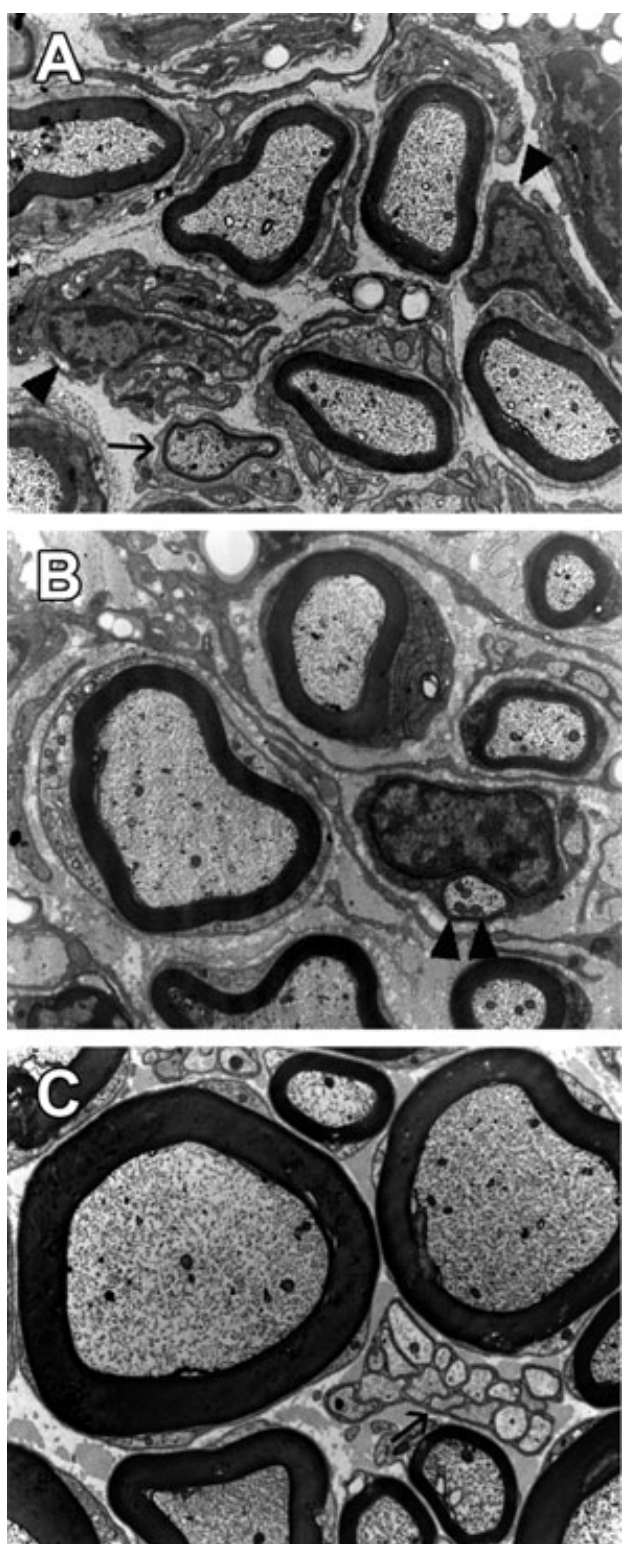

Figure 2. Ultrathin transverse sciatic nerve sections. Images correspond to sections of an area $3 \mathrm{~mm}$ distal to the crush lesion, three weeks after surgery. (A) PEMF nerve (crush lesion and treatment with a $72-\mathrm{Hz}$ electromagnetic field), showing myelinated fibers with various calibers and groups of non-myelinated fibers. Schwann cell nuclei can be observed (arrow head) and a fiber with very thin myelin sheath (arrow). (B) Control nerve (crush lesion without treatment), showing a nerve fiber in the initial process of myelinization, included in the basal membrane of a Schwann cell (double arrow head). (C) Normal nerve (without lesion or treatment), showing thick myelinated fibers and compact endoneurial space as compared to lesioned nerves and some clusters of unmyelinated fibers (double arrow). Scale bar $=2 \mu \mathrm{m}$.

density of SC nuclei was also increased in crushed nerves (PEMF and control), but there was no significant difference between them. The density and caliber of non-myelinated fibers were similar between the groups.

The analysis of oxygen-reactive species formation by the TBARS method demonstrated a significant difference between PEMF and control animals. PEMF use was associated with an increase in free-radical expression ( $p<0.05)$, which was not seen in control or normal nerves (Fig. 5). Immunohistochemical analysis for TGF- $\beta 1$, TGF- $\beta 1$ type II receptor (TGFRII), and phosphorylated Smad2/3 and Smad4 did not show differences between normal, control, and PEMF nerves (Fig. 6). Smad4 was normally seen inside nerve fibers, and TGF- $\beta 1$ surrounding it, probably corresponding to SC sites. TGF- $\beta 1$ immunoreactivity was higher in PEMF and control nerves, compared to normal ones.

\section{Discussion}

This study showed that the daily use of PEMF for three weeks after a sciatic-crush lesion failed to increase nerve regeneration and was associated with an increase in oxidative stress and a trend toward diminished myelination, neither of them influenced by TGF- $\beta 1$. However, these effects did not interfere with functional reinnervation of the paw after the lesion, denoting the lack of a direct relationship between function and intrinsic nerve condition or a diminished sensitivity of the functional test.

The effect of PEMF on blood circulation might be a factor in its possible influence on nerve regeneration. We did not find a difference between stimulated, control, and normal nerves regarding blood-vessel density or area, but did show an increase in oxygenreactive species production, which could be explained by increased circulation (Rosenspire et al., 2001; Sauer et al., 2005). This augmented concentration of free radicals could lead to SC DNA lesion (Delimaris et al., 2006) and the delayed myelination provoked by PEMF exposure. These results differ from those of Greenough (Greenough, 1992), who demonstrated an increase in blood-vessel caliber in the rabbit after PEMF exposure.

Other authors also found that exposure to PEMF did not influence regeneration. Sisken et al. (1995) found no association between PEMF use and the effects on axoplasmatic transport, a plausible way of influencing regeneration. Günay et al. (2005) submitted rats with crushed sciatic nerves to PEMF with 1, 10, 40, and $100 \mathrm{~Hz}, 1.5 \mathrm{mT}$ amplitude, for $1.5 \mathrm{~h}$ per day for 5 , 15,25 , and 38 days, and were unable to demonstrate differences from the exposure, as assessed by histological and compound action potential methods. 

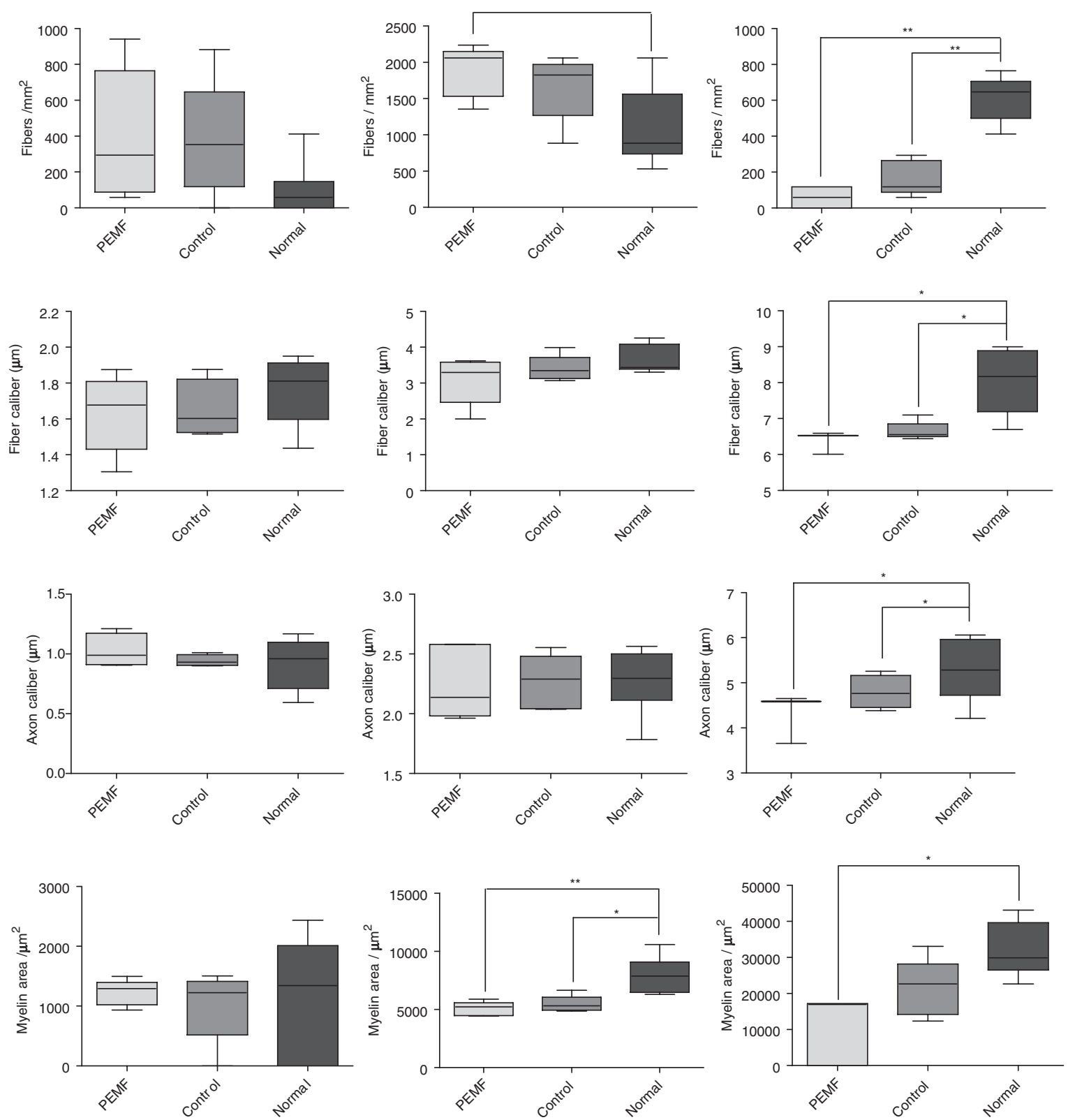

Figure 3. Morphometric data from myelinated fibers. Myelinated fibers were stratified by ranges to the morphometric analysis. The first column $(A, D, G, J)$ represents the $0-2 \mu \mathrm{m}$, the second $(B, E, H, K)$ the $2-6 \mu m$, and the third $(C, F, I, L)$ the 6-12 $\mu \mathrm{m}$ diameter. Data from density ( $A, B$, and $C$ ), fiber diameter ( $D, E$, and $F)$, axon diameter ( $G, H$, and $I)$, and myelin area $(\mathrm{J}, \mathrm{K}$, and $\mathrm{L})$ show that in the range of $0-2 \mu \mathrm{m}$ the nerves were very similar, in the $2-6 \mu \mathrm{m}$ diameter there was a trend to a bigger density of fibers in the nerves stimulated with PEMF (B) and that myelin maturation process had not reached normal values $(K)$. In the 6-12 $\mu \mathrm{m}$ range, although there are no significant differences between PEMF and control nerves, only three PEMF nerves had fibers in this range and there was a decrease on myelination in these nerves compared to normal ones, that did not happen with control nerves $(\mathrm{L})$. Data expressed in terms of median, 25-75 quartiles, minimum and maximum. ${ }^{*} \mathrm{p}<0.05,{ }^{* *} \mathrm{p}<0.01$ (Kruskal-Wallis + SNK).

Specifically, their histological results are very similar to ours, but their analysis was purely qualitative, without morphometric evaluation.

If PEMF exposure can lead to deleterious effects on the peripheral nerve regeneration process, one of the main candidates to mediate this effect should be TGF- $\beta 1$. This cytokine is synthesized by dorsal root ganglion neurons, peripheral nerves after lesion, and various immune cells, stimulating the proliferation of $\mathrm{SC}$ if they are not in contact with neurons (Rogister 


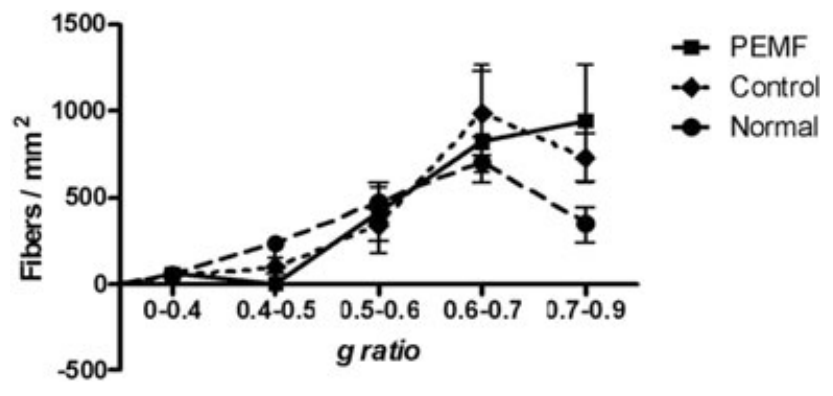

Figure 4. $g$ ratio stratified by ranges. g coefficient was obtained dividing axon diameter by fiber diameter. Higher portions of each range were included and lower ones excluded. Note that while normal (without lesion or PEMF stimulation) and control (crush lesion, without PEMF stimulation) nerves had their peaks in the $0.6-0.7$ range, those stimulated with PEMF presented their peak in the 0.7-0.9 range, probably indicating insufficient myelination in this group. Data are presented as medians and 25-75 quartiles.

et al., 1993). This can lead to a condition where the SC changes into a non-myelinating condition when in contact with neurons (Guénard et al., 1995; Sisken et al., 1995). Axonal sprouting is stimulated, and is fundamental to the initial stage of regeneration after lesion (Einheber et al., 1995; Guénard et al., 1995). Whereas in bone regeneration, increased concentrations of TGF- $\beta 1$ lead to more effective consolidation, in peripheral nerve regeneration its action can be very important in the initial phase of the

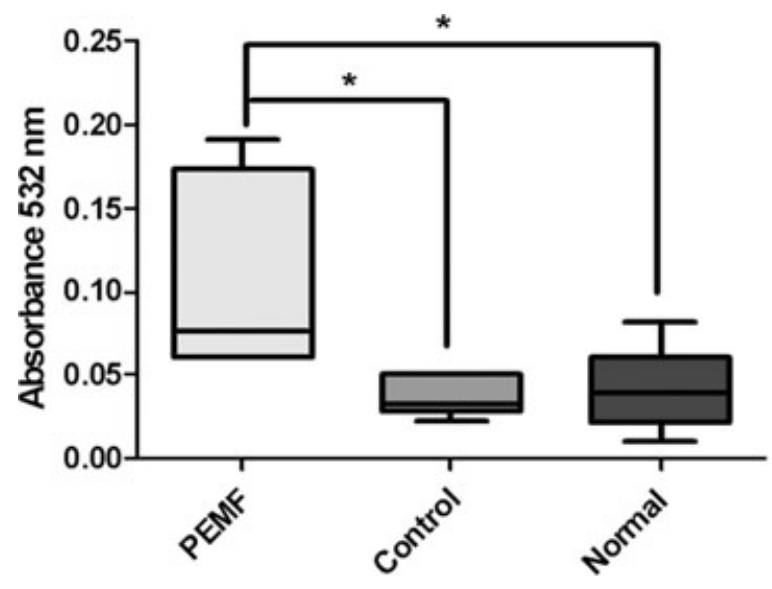

Figure 5. Analysis of reactive oxygen species formation by the TBARS (reactive substances to thiobarbituric acid) method. Figure presents results from the analysis of formation of free radicals, measured by lipidic peroxidation, using the TBARS method. There was a significant increase on oxidative stress in PEMF-stimulated nerves when compared to normal (no lesion or stimulation) and control (crush lesion, without stimulation) groups. ( ${ }^{*} p<0.05$; KruskalWallis + SNK). There was no difference between normal and control nerves. Data are expressed as medians and 25-75 quartiles.

lesion, but its maintenance can cause harmful effects, possibly by maintaining $\mathrm{SCs}$ in a non-myelinating state.

The TGF- $\beta 1$ signaling pathway includes the activation of reactive-oxygen species in various cell

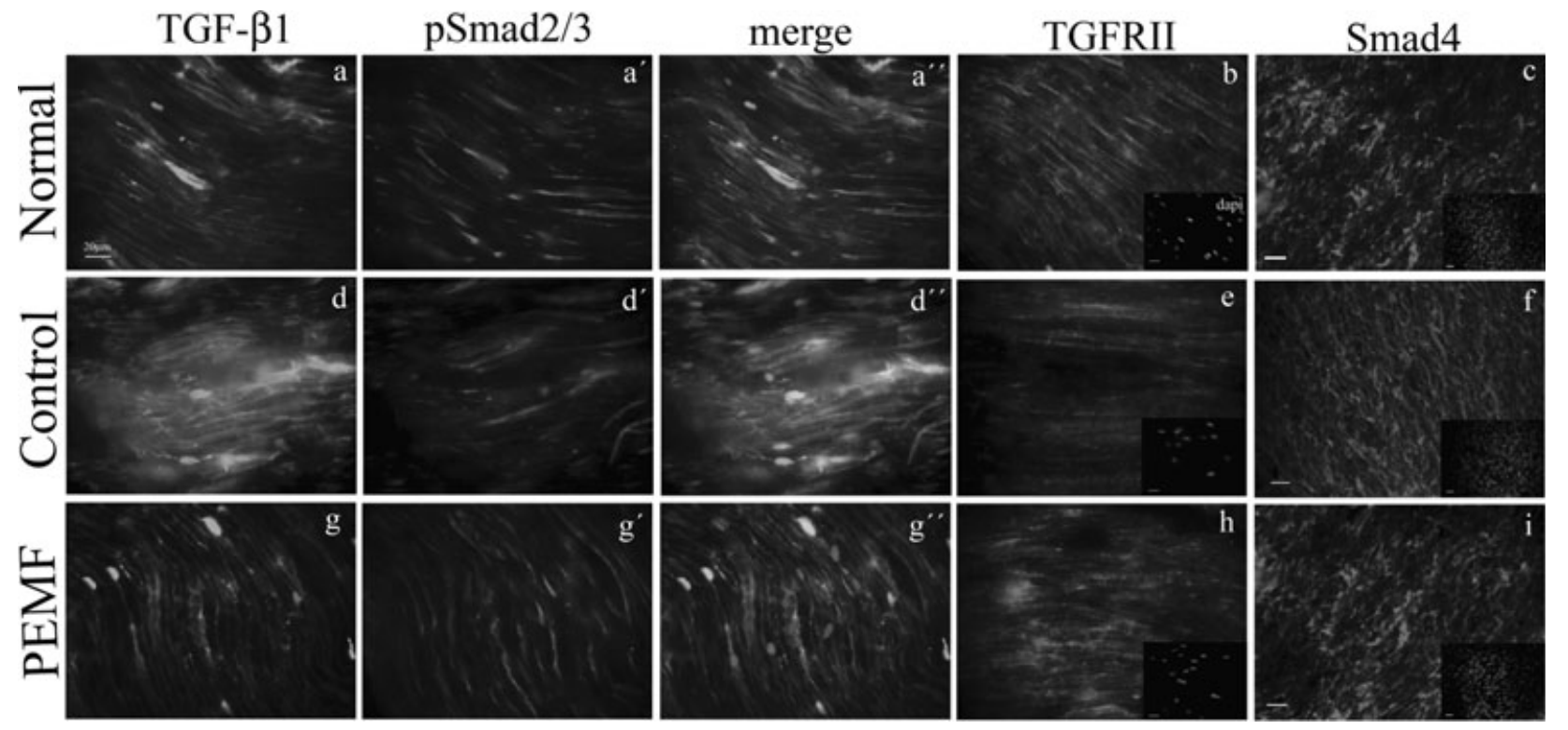

Figure 6. Longitudinal section of sciatic nerve harvested 21 days after surgery and immunostained for TGF- $\beta 1$ (a, $d$, and g), pSmad2/3 (a', d', and g'), TGF- $\beta 1$ type II receptor (TGFRII) (b, e, and h) and Smad4 (c, f, and i) from normal, control, and PEMF groups. Figures $a^{\prime \prime}, d^{\prime \prime}$, and $g^{\prime \prime}$ represent TGF- $\beta 1$ plus pSmad2/3 counterstained with DAPI, where Smad4 inside nervous fibers and TGF- $\beta 1$ surrounding it, corresponding to Schwann cells, can be observed. TGF- $\beta 1$ immunoreactivity was higher in control and PEMF groups as compared to normal animals. Scale bar $=20 \mu \mathrm{m}$. 
types (Koo et al., 1999; Junn et al., 2000; Chiu et al., 2001; Herrera et al., 2001a; 2001b; Jiang et al., 2003). In the central nervous system, TGF- $\beta 1$ is a potent neuroprotector for diverse lesions, inducing freeradical formation (Pratt and McPherson, 1997; Flanders et al., 1998; Patterson et al., 2006). PEMF stimulation leads to an increase in the TGF- $\beta 1$ expression in bone cells (Patterson et al., 2006), with previously mentioned consequences for oxidative stress. In our study, the formation of reactive-oxygen species was greater in nerves treated with PEMF than in control or normal nerves. However, immunohistochemical analysis did not show this influence, indicating that peripheral nerves may perhaps behave differently from bones when exposed to electromagnetic fields. Histological examination showed that PEMF nerves occasionally contained fibers in a very late regenerative stage, with disproportionately thin myelin sheaths in relation to their axons. Future studies could analyze this cytokine concentration in different stages of the lesion associated with PEMF use.

Although crush lesion usually favors regeneration, we showed in a previous study using a similar model that chronic transcutaneous electrical stimulation inhibits peripheral nerve regeneration (Baptista et al., 2008). We formulated the hypothesis that this effect could be related to the intensity of the electrical field generated at the site of lesion. In this study, the intensity of the electrical field was much attenuated, but the tendency to inhibit regeneration was still present. Other studies should address the same question using models of transection and suture or tubulization, in order to assess the effects of the same regimen of PEMF in a situation where regeneration is more prolonged and difficult, but has greater similarity to problematic clinical pictures in humans.

\section{Acknowledgements}

This research was supported by FBDC, FAPESB, FAPERJ and CNPq. We would like to thank Dr. Glen Gordon for supplying the PEMF dispositive for the study.

\section{References}

Balcavage WX, Alvager T, Swez J, Goff CW, Fox MT, Afdullyava S, King MW (1996). A mechanism for action of extremely low frequency electromagnetic fields on biological systems. Biochem Biophys Res Commun 222:374-378.

Baptista AF, Gomes JRS, Oliveira JT, Santos SMG, VannierSantos MA, Martinez AMB (2007). A new approach to assess function after sciatic nerve lesion in the mouse-adaptation of the Sciatic Static Index. J Neurosci Methods 161:259-264.
Baptista AF, Gomes JR, Oliveira JT, Santos SM, VannierSantos MA, Martinez AM (2008). High- and low-frequency transcutaneous electrical nerve stimulation delay sciatic nerve regeneration after crush lesion in the mouse. J Peripher Nerv Syst 13:71-80.

Bervar M (2005). Effect of weak, interrupted sinusoidal low frequency magnetic field on neural regeneration in rats: Functional evaluation. Bioelectromagnetics 26:351-356.

Chiu C, Maddock DA, Zhang Q, Souza KP, Townsend AR, Wan $Y$ (2001). TGF- $\beta$-induced p38 activation is mediated by Rac1-regulated generation of reactive oxygen species in cultured human keratinocites. Int J Mol Med 8:251-255.

Delimaris J, Tsilimigaki S, Messini-Nicolaki N, Ziros E, Piperakis SM (2006). Effects of pulsed electric fields on DNA of human lymphocytes. Cell Biol Toxicol 22:409-415.

Einheber S, Hannocks MJ, Metz CN, Rifkin DB (1995). Transforming growth factor- $\beta 1$ regulates axon/Schwann cell interactions. J Cell Biol 129:443-458.

Flanders KC, Ren RF, Lippa CF (1998). Trasnforming growth facto-betas in neurodegenerative disease. Prog Neurobiol 54:71-85.

Greenough CG (1992). The effects of pulsed electromagnetic fields on blood vessel growth in the rabbit ear chamber. $J$ Orthop Res 10:256-262.

Guénard V, Gwynn LA, Wood PM (1995). Tranforming growth factor- $\beta$ blocks myelination but not ensheathment of axons by Schwann cells in vitro. J Neurosci 15:416-428.

Günay MGI, Özgünen K, Zorludemir S (2005). Effect of pulsed magnetic field on regenerating rat sciatic nerve: an in-vitro electrophysiological study. Int J Neurosci 115:881-892.

Herrera B, Alvarez AM, Sánchez A, Fernández M, Roncero C, Benito M, Fabregat I (2001a). Reactive oxygen species mediates the mitochondrial dependent apoptosis induced by transforming growth factor (beta) in fetal hepatocytes. FASEB J 15:741-751.

Herrera B, Fernández M, Roncero C, Ventura JJ, Porras A, Valladares A, Benito M, Fabregat I (2001b). Activation of p38MAPK by TGF- $\beta$ in fetal rat hepatocytes requires radical oxygen production, but is dispensable for cell death. FEBS Lett 499:225-229.

Hong YH, Peng HB, La Fata V, Liao JK (1997). Hydrogen peroxide-mediated transcriptional induction of macrophage colony-stimulator factor by TGF- $\beta 1$. J Immunol 159:2418-2423.

Jiang Z, Seo JY, Ha H, Lee EA, Kim YS, Han DC, Un ST, Park CS, Lee HB (2003). Reactive oxygen species mediate TGF$\beta 1$-induced plasminogen activator inhibitor-1 upregulation in mesangial cells. Biochem Biophys Res Commun 309:961-966.

Junn E, Lee KN, Ju HR, Han, SH, Im JY, Kang HS, Lee TH, Bae YS, Ha KS, Lee ZW, Rhee SG, Choi I (2000). Requirement of hydrogen peroxide generation in TGF- $\beta 1$ signal transduction inhuman lung fibroblast cells: Involvement of hydrogen peroxide and Ca2+ in TGF- $\beta 1$ induced IL-6 expression. J Immunol 165:2190-2197.

Kanje M, Rusovan A, Sisken B, Lundborg G (1993). Pretreatment of rats with pulsed electromagnetic fields enhances regeneration of the sciatic nerve. Bioelectromagnetics 14:353-359.

Kim SS, Shin HJ, Eom DW, Huh JR, Woo Y, Kim H, Ryu SH, Suh PG, Kim MJ, Kim JY, Koo TW, Cho YH, Chung SM (2002). Enhanced expression of neuronal nitric oxide synthase and phospholipase C-gamma1 in regenerating 
murine neuronal cells by pulsed electromagnetic field. Exp Mol Med 34:53-59.

Koo HY, Shin I, Lee ZW, Lee SH, Kim SH, Lee CH, Kang HS, Has KS (1999). Roles of RhoA and phospholipase $A 2$ in the elevation of intracellular $\mathrm{H}_{2} \mathrm{O}_{2}$ by transforming growth factor-beta in Swiss 3T3 fibroblasts. Cell Signal 11:677-683.

Lafon C, Mathieu C, Guerrin M, Pierre O, Vidal S, Valette A (1996). Transforming growth factor beta 1-induced apoptosis in human ovarian carcinoma cells: protection by antioxidant N-acetylcysteine and bcl-2. Cell Growth Differ 7:1095-1194.

Macias MY, Battocletti JH, Sutton CH, Pintar FA, Maiman DJ (2000). Directed and enhanced neurite growth with pulsed magnetic field stimulation. Bioeletromagnetics 21:272-286.

Menezes D, Valentim C, Oliveira MF, Vannier-Santos MA (2006). Putrescine analogue cytotoxicity against Trypanosoma cruzi. Parasitol Res 98:99-105.

Oschman JL (2004). Recent developments in bioelectromagnetic medicine. In: Bioelectromagnetic Medicine. Rosch PJ, Markov MS (Eds). Informa Health Care, London, pp 77-92.

Ottani V, De Pasquale V, Govoni P, Franchi M, Zaniol P, Ruggeri $A$ (1998). Effects of pulsed extremely-low-frequency magnetic fields on skin wounds in the rat. Bioelectromagnetics 9:53-62.

Patterson TE, Sakai Y, Grabiner MD, Ibiwoye M, Midura RJ, Zborovski M, Wolfman A (2006). Exposure of murine cells to pulsed electromagnetic fields rapidly activates the mTOR signaling pathway. Bioelectromagnetics 27:535-544.

Pratt BM, Mcpherson JM (1997). TGF-beta in the central nervous system: potential roles in ischemic injury and neurodegenerative diseases. Cytokine Growth Factor Rev 8:267-292.

Rhyu DY, Yang Y, Ha H, Lee GT, Song JS, Uh ST, Lee HB (2005). Role of reactive oxygen species in TGF- $\beta 1$-induced mitogen-activated protein kinase activation and epithelial- mesenchymal transition in renal tubular epithelial cells. J Am Soc Nephrol 16:667-675.

Rogister B, Delrée P, Leprince P, Martin D, Sadzot C, Malgrange B, Munaut C, Rigo JM, Lefebvre PP, Octave JN, Schoenen J, Moonen G (1993). Transforming growth factor beta as a neurononglial signal during peripheral nervous system response do injury. J Neurosci Res 34:32-43.

Rosenspire AJ, Kindzelskii AL, Petti HR (2001). Pulsed DC electric fields couple to natural $\mathrm{NAD}(\mathrm{P}) \mathrm{H}$ oscillations in $\mathrm{HT}$ 1080 fibrosarcoma cells. J Cell Sci 114:1515-1520.

Rusovan A, Kanje M, Mild KH (1992). The stimulatory effect of magnetic fields on regeneration of the rat sciatic nerve is frequency dependent. Exp Neurol 117:81-84.

Sauer H, Bekhite MM, Hescheler J, Wartenberg M (2005). Redox control of angiogenic factors and CD31-positive vessel-like structures in mouse embryonic stem cells after direct current electric field stimulation. Exp Cell Res 304:380-390.

Shin DM, Jeon JH, Kim CW, Cho SY, Lee HJ, Jang GY, Jeong EM, Lee DS, Kang JH, Melino G, Park SC, Kim IG (2008). TGF beta mediates activation of transglutaminase 2 in response to oxidative stress that leads to protein aggregation. FASEB J 22:1-10

Sisken BF, Kanje M, Lundborg G, Herbest E, Kutz W (1989). Stimulation of rat sciatic nerve regeneration with pulsed electromagnetic fields. Brain Res 485:309-316.

Sisken BF, Jacob JM, Walker JL (1995). Acute treatment with pulsed electromagnetic fields and its effects on fast axonal transport in normal and regenerating nerve. J Neurosci Res 42:692-699.

Vannier-Santos MA, Menezes D, Oliveira MF, de Mello FG (2008). The putrescine analogue 1,4-diamino-2-butanone affects polyamine synthesis, transport, ultrastructure and intracellular survival in Leishmania amazonensis. Microbiology 154:3104-3111. 